\title{
Estructura del plan de crisis
}

\author{
Dra. Ma Luisa Sánchez Calero \\ Profesora de Periodismo Especializado \\ Universidad Carlos III, Madrid
}

\section{RESUMEN}

Son muchos los factores que tenemos que tener en cuenta para prevenir una situación de crisis o para actuar cuando la situación ya ha estallado. Es importante conocer cuando y cómo se produce la crisis, la tipología de las crisis que estamos predispuestos a padecer, la formación del comité de crisis, la gestión de la comunicación de crisis, la actitud de los órganos de representación sindical o la estructura del plan de crisis.

Este artículo sigue la misma línea de investigación que presenté en el trabajo publicado en el número anterior de la Revista. Donde abordaba otro aspecto de este tema que llevaba por título "Importancia de la Gestión de la Comunicación en periodos de Crisis Empresariales". En la misma línea, este otro capítulo expone de forma clara y objetiva cómo debe ser estructurado un Plan de Crisis desde la propia Compañía.

\section{ABSTRACT}

There are many factors that we have to take into account to avoid a critical situation or to act when the situation has already taken place. There is importand to know when and how the crisis was produced, the type of crisis we are liable to suffer, the formation of the crisis commitee. The negociations of the communication of the crisis, the administering of the communication of the crisis, the attitude of the trade union representatives or structure of crisis plan.

This articule follows the line of investigation that I published the last edition of this review. Where I explained another aspect of the crisis that was titled "the importance of the administering of the communication in moments of crisis in the companies"

Palabras claves: Plan de Crisis/Manual de crisis/Tipología de la crisis/Situaciones de crisis.

Key words: Plan of crisis/Document of crisis/The type of crisis/Critical situation.

\section{Bases de un plan de crisis}

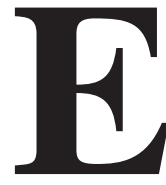

1 plan diseñado para aminorar los efectos que produce la crisis en cualquier organización comporta una serie de medidas de sentido común. Cada una de estas medidas deben de responder a cuestiones tan elementales como: ¿Cuáles son los proveedores o servicios externos que pueden ser necesarios?. ¿Qué papel debe ocupar el departamento de Comunicación 
ante la crisis?. ¿Cómo se puede contactar con los directivos fuera de la jornada habitual?. ¿Está la centralita del teléfono equipada para recibir gran número de llamadas?, etc.

En general se puede señalar que todo plan de crisis debe contener las actividades principales a seguir antes, durante y después de finalizada la crisis. En este sentido, el documento debe recoger cada una de las supuestas crisis a las que por su tipo de actividad puede verse inmersa la organización y analizar los recursos humanos y económicos con los que se cuenta y prever además las respuestas a cuatro apartados fundamentales según nuestro criterio:

a) Definir el objetivo que hay que lograr en cada caso.

b) Elaborar el mensaje que habrá que emitir en consecuencia.

c) Definir las audiencias y los canales de comunicación oportunos.

d) Preparar un plan de actuación. En este plan de actuación es menester no olvidar algunas reglas básicas como son: Contar con la máxima información sobre la empresa. Identificar todos los aspectos positivos con los que cuenta la empresa. Y definir aspectos menores pero importantes en cada caso de crisis.

En esta última etapa descrita, el plan de actuación debe de recoger también una serie de relacionadas con situaciones que pueden generar una situación concreta según el tipo de empresa. Y las actividades son básicamente las siguientes:

a) Actividades encaminadas a descubrir señales que puedan ser motivo de alarma dentro de la empresa o escenarios que se puedan dar en cada tipo de crisis.

b) Identificar los procesos, políticas, públicos y expertos que puedan participar en la empresa en caso de producirse la crisis.

c) Diseñar cada una de las áreas y sus respectivas responsabilidades.

d) Determinar el presupuesto necesario para llevar a cabo las tareas de simulación y puesta a punto del plan.

Este plan de crisis tiene que poseer además tres normas fundamentales para que se convierta en eficaz.

a) La primera es que "todo plan debe ser comprobado y ensayado". Para ello es importante poseer un esquema operativo que evite la improvisación ante sus públicos. Un ejemplo que ilustra esta situación lo hemos recogido en la Institución del Liceo Francés de Madrid, donde el primer día de cada nuevo curso se les explica al alumnado el significado de cada uno de los toques de la campana. Uno de esos toques es el de alarma y se efectúa una prueba durante todos los trimestres del curso. Cuando es emitido su sonido los casi cuatro mil alumnos del colegio se presentan a toda velocidad en el campo de rugby (una de las zonas más 
seguras) del colegio. Ante esta situación de prevención no cabe duda que si algún día sucede algo imprevisto sus efectos se habrán aminorado al máximo.

b) La segunda norma del Plan de Crisis es la de "actualizarlo periódicamente”. La situación ideal sería actualizarlo con carácter trimestral con el objetivo de tener al día todos los apartados; especialmente el de la lista de prensa, ante los frecuentes cambios de los periodistas. Pero esta es una medida muy lejana aún para las escasas empresas que poseen su plan de crisis. Un ejemplo a seguir lo tenemos en la compañía Iberia; una de las pocas empresas españolas que dispone de este manual y lo actualiza anualmente.

c) Otro aspecto importante a la hora de elaborar el Plan de Crisis es "la incidencia del coste económico". En este sentido hay que recordar que en la empresa sucede como en la vida real, cuando las cosas funcionan en lo último que se piensa es en que hay que destinar un presupuesto para el Plan de Crisis. Y por otro lado, en épocas de "vacas flacas" cuando los beneficios empresariales decrecen, la planificación de la crisis sigue siendo un candidato perfecto a ser eliminado del presupuesto. ${ }^{1}$

A pesar de lo apuntado el problema de esta situación radica en que la dirección siempre preocupada por el coste de las cosas son reacios a dedicar una gran cantidad de recursos al desarrollo de un plan para algo cuyo alcance y dimensión son desconocidos, como en el caso de cualquier desastre. Y ello es debido a que el Plan de Crisis precisa de cometidos logísticos y operativos que requieren de un alto coste económico.

Para evitar por lo tanto esta situación nuestra propuesta según se desprende de la investigación seguida consiste en concienciar a la empresa de la efectividad del mismo atendiendo a dos puntos principalmente a lo largo del proceso de planificación:

a) Mantener los costes al mínimo mediante una planificación que conlleve acciones adecuadas en relación coste-efectividad.

b) Mantener al mínimo las acciones del Plan de Crisis.

En cualquier caso, la empresa debe tratar de obtener una solución que le permita contar con una serie de medidas preventivas en caso de que se produzca una situación de crisis. Y como medida principal a tener en cuenta es la de la responsabilidad social de la organización; por encima de cualquier objetivo eco-

1 Entrevista mantenida en el 2002 con D. Carlos Paniagua, Asesor experto en Programas de Crisis. 
nómico. De tal forma que la protección de las propiedades y activos de la empresa quede subordinada a la protección de los trabajadores, de sus clientes y de la propia comunidad en la que reside.

Este trabajo de prevención ante la crisis requiere de una gran elaboración y preparación de la información adecuada. Pero es indudable según se ha experimentado en empresas que actualmente lo poseen y lo han ejecutado que permite:

a) Poner en marcha inmediatamente un servicio "ad hoc" como la mejor fuente de información. Esto significa comenzar a ganar la batalla de la anticipación disponiendo de una serie de documentos principales.

b) Controlar la orientación de las informaciones que van a entrar en circulación. La agilidad para controlar la información de las primeras veinticuatro horas suele ser de vital importancia, sobre todo en lo que se refiere a inducir ciertas posiciones de los medios de comunicación que puedan determinar la postura frente al conflicto.

c) Dar prueba del sentido de la responsabilidad. Una responsabilidad que en nuestro criterio va más allá de lo puramente físico porque en esta situación es importante controlar la información que se da y como se proporciona. Un consejo en estos casos que comparten muchos profesionales, es proporcionar una comunicación mínima, discreta y transparente.

Pauchan y Mitroff (1992) advierten que en las empresas americanas son cuatro las categorías principales sobre las que la compañía toma mayores medidas de prevención:

a) Actividades cuyas finalidades consistan en comprobar si la empresa toma las medidas oportunas en la utilización de materiales peligrosos, sistemas legales o financieros o si se adapta en materia de seguridad a la legislación vigente.

b) Modificación o estructuración interna destinadas a la seguridad de los productos e instalaciones.

c) Formación a los empleados y directivos de la organización a que sepan como enfrentarse emocionalmente ante una situación de crisis.

d) Comunicarse con sus públicos externos para obtener información certera acerca del entorno.

\section{El manual de crisis de una organización y sus medidas principales}

En la mayor parte de las empresas españolas consultadas para abordar esta investigación hemos podido comprobar que no disponen de un Manual de crisis.

No es habitual encontrar este documento y no es cuestión ni de su tamaño ni de su actividad si no simplemente es una herramienta poco utilizada porque no consideran práctica la utilidad de la misma; ya que difícilmente reconocen que han sufrido alguna crisis. 
Sin embargo, en aquellas otras compañías que disponen desde hace años de esta herramienta, como es el caso de las compañías Iberia, Alcampo o BBV, coincidían sus responsables de comunicación en señalar una serie de puntos, actividades y elementos que son prácticamente comunes en cualquier Manual de Crisis.

Entre sus recomendaciones principales advertían de unos puntos que debe de abordar cualquier Manual de Crisis, aunque luego en cada empresa según sus propias características y morfologías de crisis, serán incluidos otros contenidos:

a) Metas a alcanzar mediante la ejecución de dicho Plan de Crisis.

b) Filosofía donde se recojan los objetivos y principios básicos de la organización. En este apartado suelen estar incluido los principios, valores, normas, costumbres y principios básicos que rige a la compañía.

c) Justificación de las razones que hacen necesario la elaboración de este Manual de Crisis y de un comité de la crisis.

d) Estudio sobre los tipos de crisis potenciales a los que está expuesta la organización.

e) Normas básicas generales de actuación que sean eficaz ante una situación de crisis.

f) Listado con datos, nombres, y medios para hacer llegar la información a los medios de comunicación.

g) El centro de reunión del comité a lo largo de la crisis.

h) Guía de asociaciones, expertos, organismos oficiales, asociaciones, agrupaciones, etc., que puedan servir de ayuda complementaria.

i) Funcionamiento y puesta en marcha del Plan de Crisis mediante simulacros de crisis potenciales a las que pueda estar sometida la empresa. El objetivo de esta medida es comprobar el funcionamiento del plan o inclusión de las mejoras oportunas.

La curiosidad por este tema nos ha llevado a la búsqueda de manuales de crisis de compañías afincadas en España. Una búsqueda que en muchos momentos no ha sido fortuita y sólo en raras excepciones cuando hemos encontrado alguno, la empresa nos ha mostrado su gran recelo a que fuese publicado o simplemente analizado.

En la figura se muestra parte del índice de un Manual de Crisis facilitado por la compañía Iberia. En el podemos apreciar los temas principales que la Compañía considera esenciales para estar en posición de alerta permanente. 
Figura A. Indice Plan de Crisis

\begin{tabular}{|c|c|c|}
\hline IBERIA & PLAN DE RESPUESTA ANTE UN ACCIDENTE AEREO & $\begin{array}{c}\text { Pág.: } 2 \\
22 / 07 / 96\end{array}$ \\
\hline
\end{tabular}

\section{a. GENERALIDADES}

\subsection{INDICE}

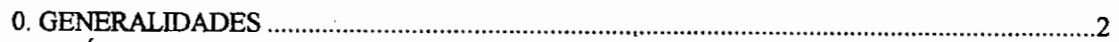

0.1. ÍNDICE

0.2. LISTA DE DISTRIBUCIÓN

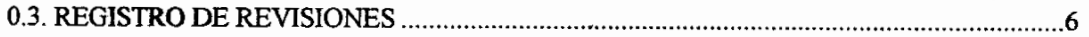

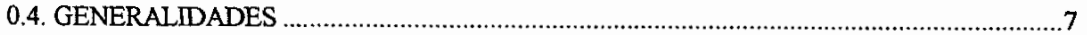

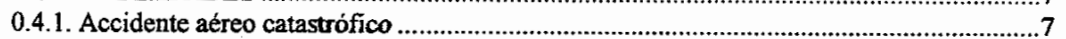

0.4.2. Respuesta ante un accidente aéreo catastrófico .......................................................... 7

0.4.3. "Plan de Repuesta ante un Accidente Aéreo" .........................................................8

1. POLITICA DE COMPAÑIA E INTRODUCCIÓN

1.1. POLÍTICA DE COMPAÑIIA

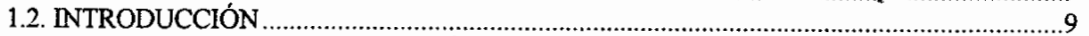

2. ANEXO 13 DE OACI - INVESTIGACIÓN DE ACCIDENTES ........................................11

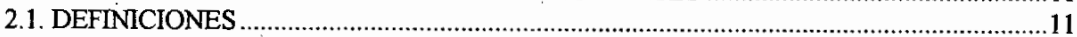

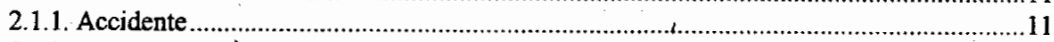

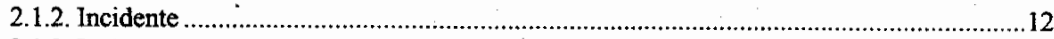

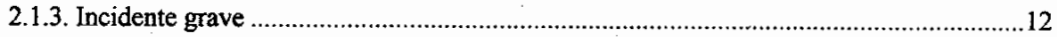

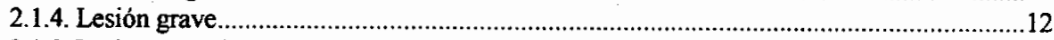

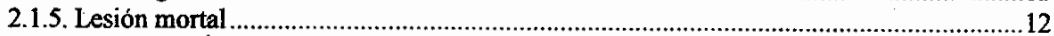

2.2. INVESTIGACIÓN DE ACCDENTES ..............................................................12

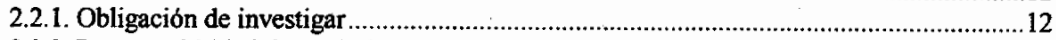

2.2.2. Responsabilidad de instituir y realizar la investigación .............................................13

2.2.3. Derecho a participar en la investigación .........................................................13

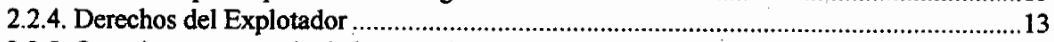

2.2.5. Organismo encargado de la Investigación Oficial .................................................13

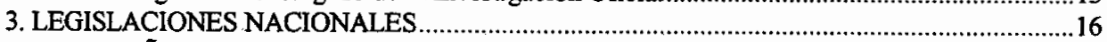

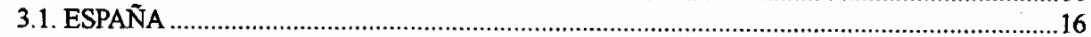

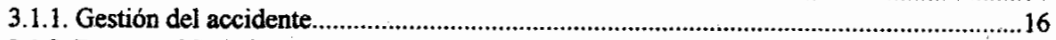

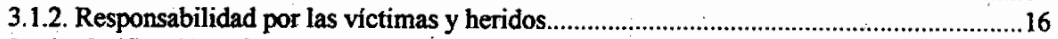

3.1.3. Notificación a familiares de víctimas y beridos.......................................................16

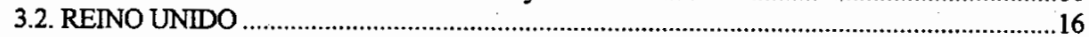

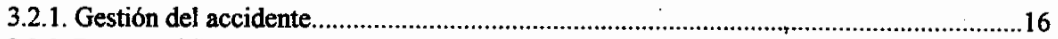

3.2.2. Responsabilidad por las víctimas y heridos........................................................... 16

3.2.3. Notificación a familiares de víctimas y heridos ..........................................................16

4. NIVEL DE RESPUESTA ANTE UNA EMERGENCIA AÉREA .....................................17

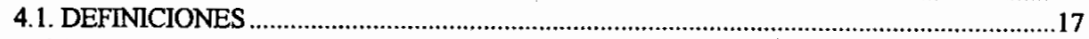

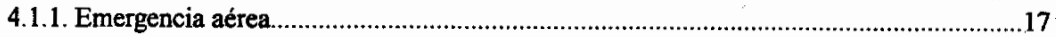

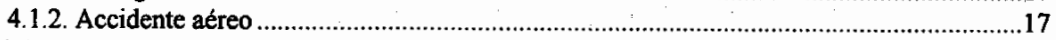

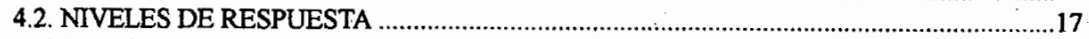

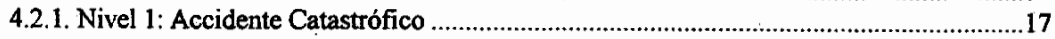

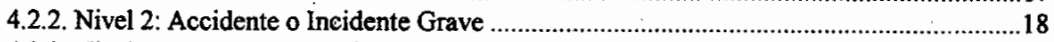

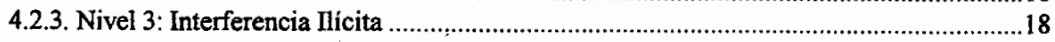


4.2.4. Nivel 4: Accidente sufrido por otras compañias (Participada, Código Compartido, etc) 18

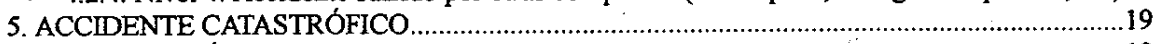

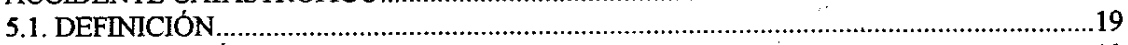

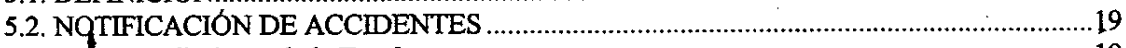

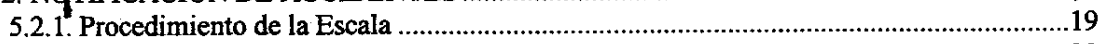

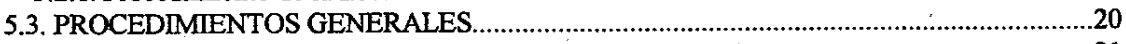

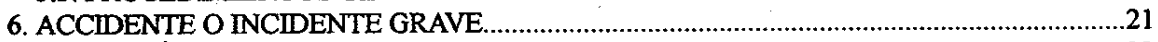

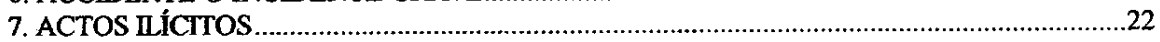

7.1. AMENAZA DE EXPLOSIVO A BORDO

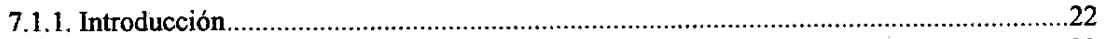

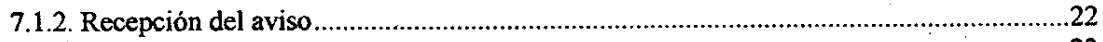

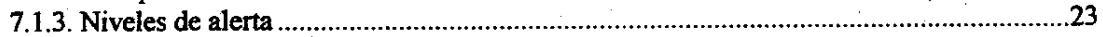

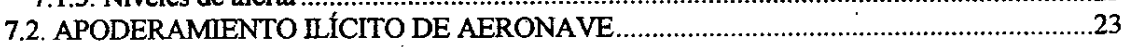

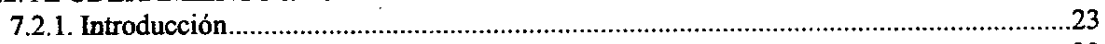

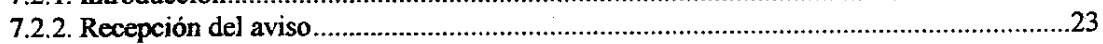

7.2.3. Respuesta de las Autoridades del Aeropuerto ..........................................................24

8. ACCIDENTES OTRAS COMPAÑ́́AS (PARTICIPADAS, CÓDIGO COMPARTIDO, ETC.) ....25

- 9. CENTRO DE CONTROL DE EMERGENCIAS AERONÁUTICAS ...........................................26

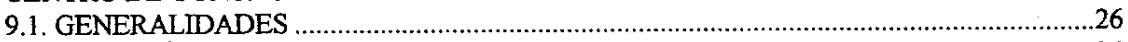

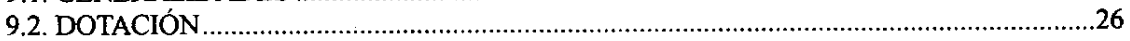

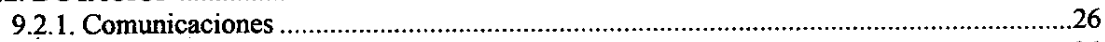

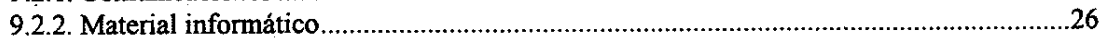

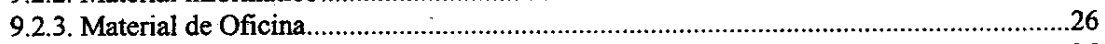

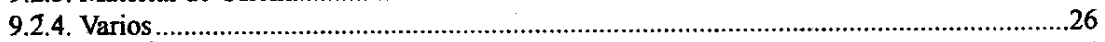

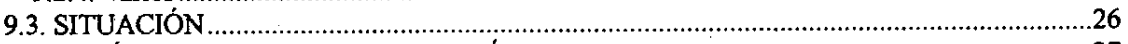

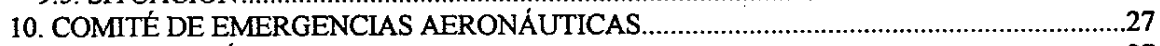

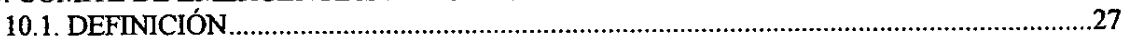

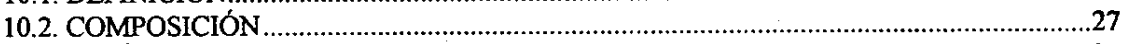

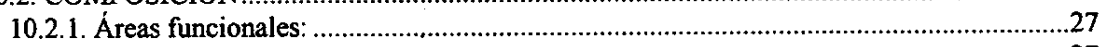

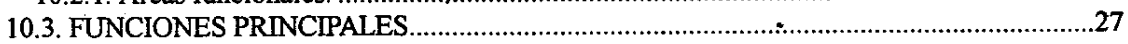

10.3.1. Presidente del "Comité de Emergencia “...................................................................2

10.3.2. Coordinador Central ................................................................................................28

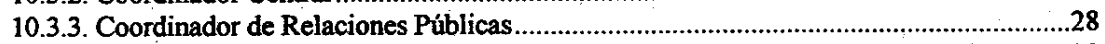

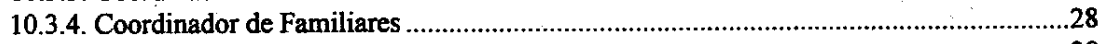

10.3.5. Coordinador de Gestión de Riesgos ...................................................................28

10.4. MIEMBROS PERMANENTES DEL COMTTÉ

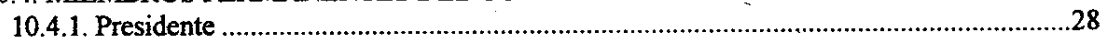

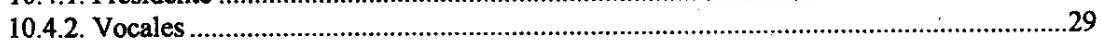

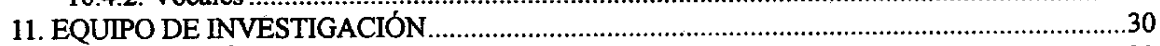

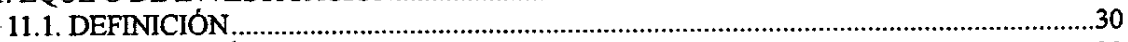

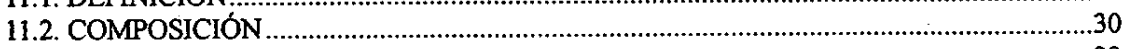

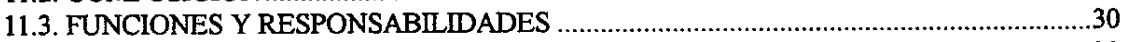

11.3.1. Jefe Equipo de Investigación .................................................................................30

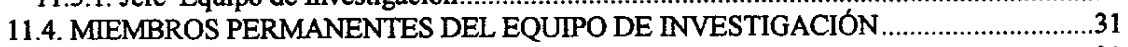

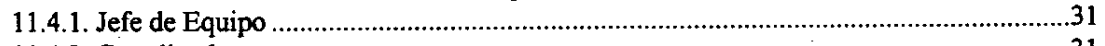

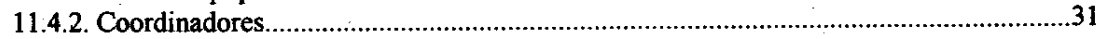




\begin{tabular}{|c|c|c|}
\hline IBERIA & PLAN DE RESPUESTA ANTE UN ACCIDENTE AEREO & Pág.: 4 \\
$22 / 07 / 96$ \\
\hline
\end{tabular}

12. EQUIPO AUXILIAR (COORDINADORES BIENESTAR SOCIAL) ……...........................32

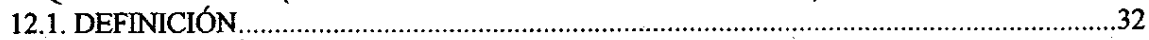

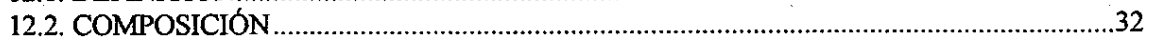

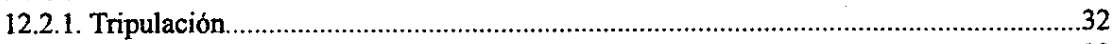

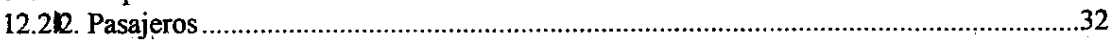

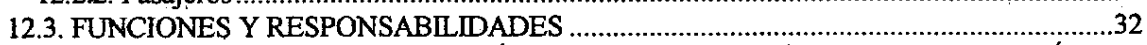

13. CENTRO LOCAL DE INFORMACIÓN Y CENTROS LOCALES DE ATENCIÓN AL

PASAJERO.

14. PROCEDIMIENTOS PARA RECLAMACIONES .....................................................................

15. RELACIONES PÚBLICAS Y ACTUACIONES ANTE LOS MEDIOS DE COMUNICACIÓN35

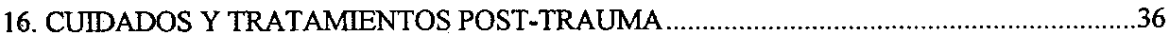

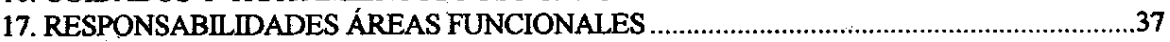

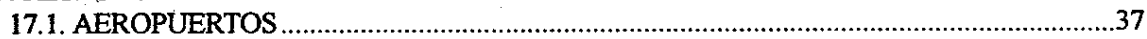

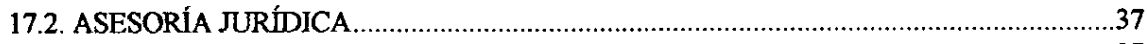

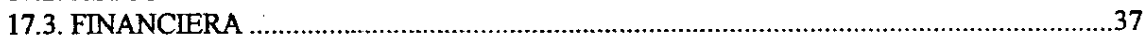

17.4. GABINETE DE PRENSA

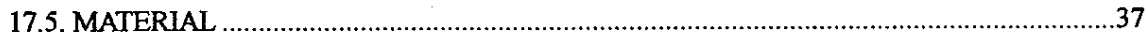

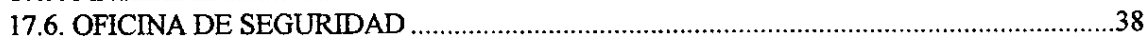

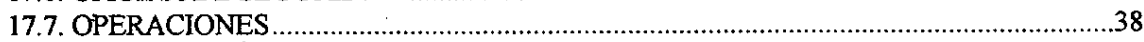

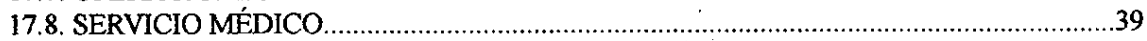

17.9. SISTEMAS

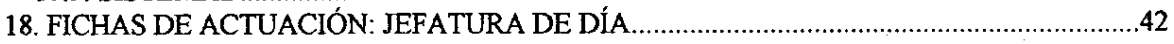

18.1. DETERMINAR NIVEL DE RESPUESTA APLICABLE ...............................................42

18.2. ACCIONES NIVEL 1- ACCIDENTE CATASTRÓFICO -............................................4

18.3. ACCIONES NIVEL 2 - ACCIDENTE O INCIDENTE GRAVE - ................................44

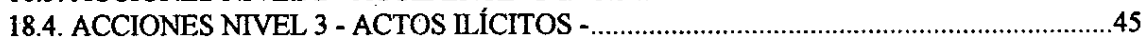

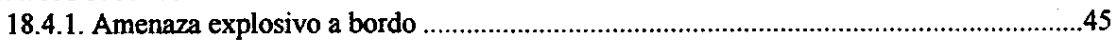

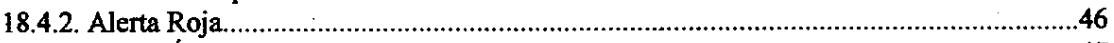

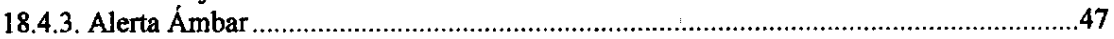

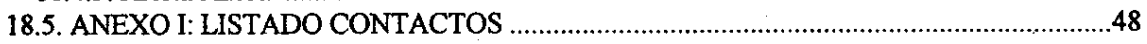

18.5.1. Miembros del Comité de Emergencias Aeronáuticas .....................................................48

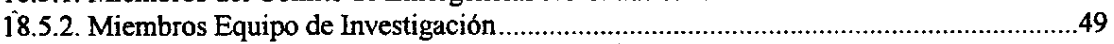

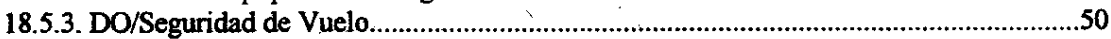

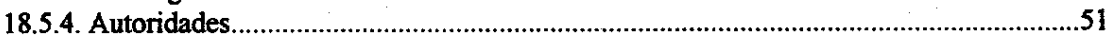

18.5.5. Embajadas y Consulados ....................................................................................5

18.6. ANEXO 2: ACCIONES INICIALES ÁREA OPERACIONES …...................................5

18.7. ANEXO 3: ACCIONES INICIALES ÁREA MATERIAL ….........................................5

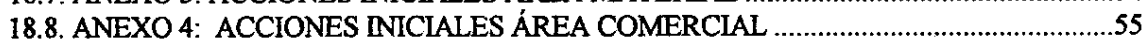

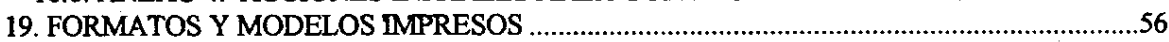

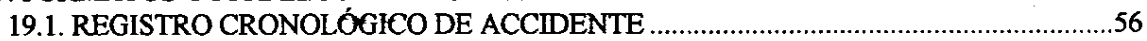

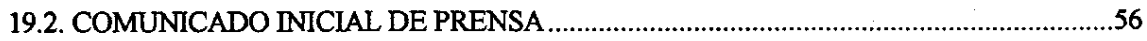

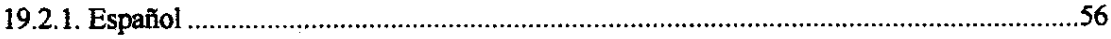

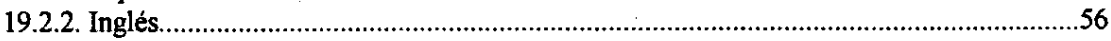

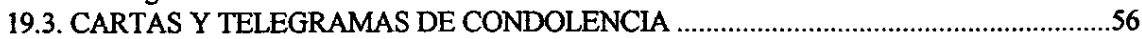




\section{Bibliografía:}

- CARRASCOSA, J. Luis.: Comunicación: Una comunicación eficaz para el éxito de los negocios. Ciencias de la Dirección, Madrid 1992.

- FINK, S.: Crisis management: Planning for the inevitable. Amacon (American Management Association): Nueva York. 1986.

- HEARLE, D.G. Planning for crisis. En J.Gottschall (Ed). Crisis response. Inside stories on managing image under siege. Gale Research, Inc.: Detroit, Michigan. (1993).

- KAUFMAN, A. El poder de las organizaciones. Ed. ESIC. Madrid 1993.

- LUCAS MARÍN, A. La comunicación en la empresa y en las organizaciones. Bosch ed. Barcelona 1997.

- MITROFF, I.,Pauchant, T.C. y Shrivastava, P. Conceptual and empirical issues in the development for a general theory of crisis management. Technological Forecasting and Social Change, vol. 33. (1988).

- Otras bibliografías.: Gran parte de esta investigación se ha realizado mediante la documentación extraída a través de entrevistas personales realizadas a Directores de Comunicación de compañías, Portavoces en situaciones de crisis, Miembros del comité de crisis, Secretarias, Asesores externos, etc. 